\title{
Predictive Process Adjustment by Detecting System Status of Vacuum Gripper in Real Time during Pick-Up Operations
}

\author{
Sujeong Baek* and Dong Oh Kim
}

Citation: Baek, S.; Kim, D.O

Predictive Process Adjustment by Detecting System Status of Vacuum Gripper in Real Time during Pick-Up Operations. Processes 2021, 9, 634. https://doi.org/10.3390/pr9040634

Academic Editor: Luis Puigjaner

Received: 9 March 2021

Accepted: 1 April 2021

Published: 5 April 2021

Publisher's Note: MDPI stays neutral with regard to jurisdictional claims in published maps and institutional affiliations.

Copyright: (c) 2021 by the authors. Licensee MDPI, Basel, Switzerland. This article is an open access article distributed under the terms and conditions of the Creative Commons Attribution (CC BY) license (https:/ / creativecommons.org/licenses/by/ $4.0 /)$.
Department of Industrial Management Engineering, Hanbat National University, 125 Dongseo-Daero, Yuseong-Gu, Daejeon 34158, Korea; 20151470@edu.hanbat.ac.kr

* Correspondence: sbaek@hanbat.ac.kr; Tel.: +82-42-821-1228

\begin{abstract}
In manufacturing systems, pick-up operations by vacuum grippers may fail owing to manufacturing errors in an object's surface that are within the allowable tolerance limits. In such situations, manual interference is required to resume system operation, which results in considerable loss of time as well as economic losses. Although vacuum grippers have many advantages and are widely used in the industry, it is highly difficult to directly monitor the current machine status and provide appropriate recovery feedback for stable operation. Therefore, this paper proposes a method to detect the success or failure of a suction operation in advance by analyzing the amount of outlet air pressure in the Venturi line. This was achieved by installing an air pressure sensor on the Venturi line to predict whether the current suction action will be successful. Through empirical experiments, it was found that downward movements in the z-axis of the vacuum gripper can easily rectify a faulty gripper suction operation. Real-time monitoring results verified that predictive process adjustment of the pick-up operation can be performed by modifying the z-position of the vacuum gripper.
\end{abstract}

Keywords: real-time monitoring; fault detection; predictive process adjustment; vacuum gripper; sensor data

\section{Introduction}

\subsection{Theoretical Background}

In industrial production, as manufacturing systems have become more complicated, the concept of operations and maintenance (O\&M) has become important in preventing unforeseen faults and errors and for smooth operations [1,2]. Lin et al. (2021) found that $O \& M$ investments are continuously increasing in the power plant industry owing to concerns regarding the safe and reliable operation of power plants [3]. Accordingly, the authors investigated the major influencing factors in the O\&M of power plants by constructing a fishbone diagram and analyzing the gray correlation between the derived factors. In addition, O\&M is relevant not only to physical products or systems but to cyber systems as well. In this regard, Furumoto et al. (2020) discussed how to assess and prevent cyber risks to achieve reliable ship operation [4]. In manufacturing processes, the prevention of unknown and sudden faults in real time is critical [5,6].

Grippers play an important role in an industrial manufacturing system [7-9]. They can be classified into two types: mechanical type and vacuum type. Mechanical grippers are also known as robotic grippers [10]. As their shape and operating mode mimic human hands and fingers, they are usually used for performing complex or delicate operations. For example, Vedhagiri et al. (2019) proposed a mechanical gripper with five fingers in order to handle objects with complex shapes, such as a coin, cosmetic cream, and fruit [11]. A mechanical gripper with three fingers has also been proposed to conduct micromanipulation [12]. The authors [12] applied a piezoceramic transducer for effectively handling objects as small as 10 to $800 \mu \mathrm{m}$, such as glass hollow microspheres and iron spheres. 
On the other hand, vacuum grippers utilize suction cup(s), air pipe(s), and Venturi line(s) to handle objects, and they require an external air compressor to supply compressed air, as illustrated in Figure 1. In particular, a discrete manufacturing process is typically performed by several common operations using vacuum grippers, and the grippers are widely used for lifting, transporting, and inspections [13-15] as they are simple, inexpensive to install, easy to operate with fragile objects, and can handle various types of object shapes $[16,17]$.

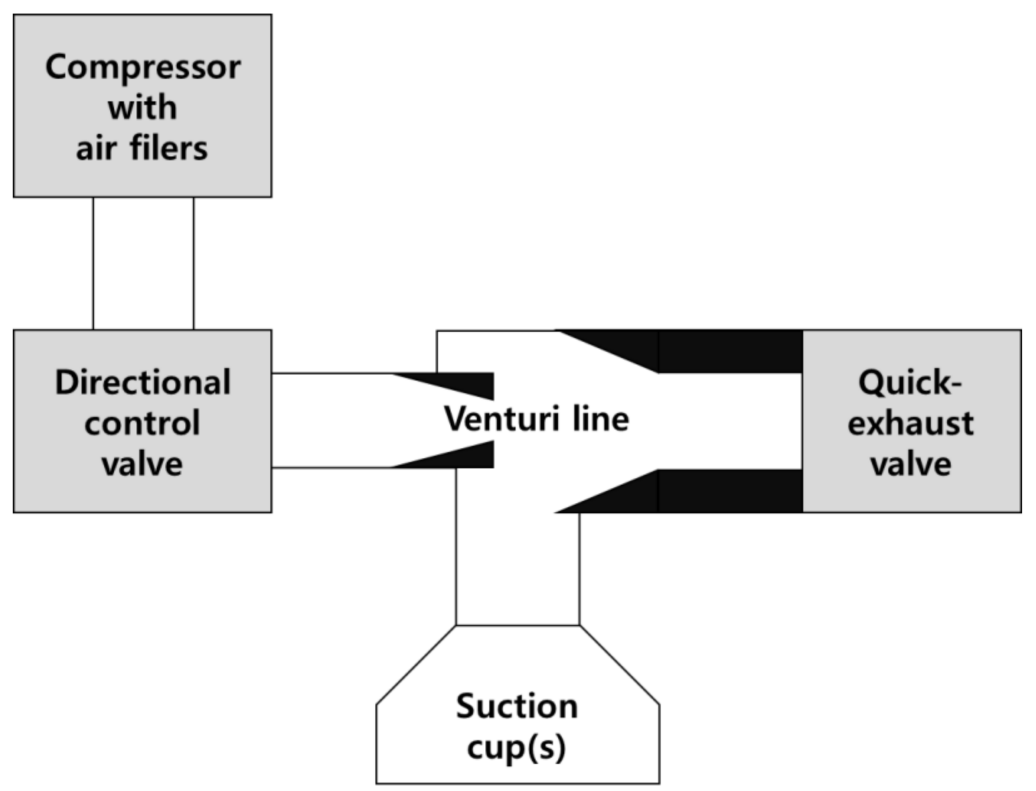

Figure 1. Illustration of a vacuum gripper system (composed with a suction cup and the connected Venturi line).

For example, a vacuum gripper with four suction cups placed in a two by two arrangement has been proposed for handling heavy objects with complex shapes [18]. Amend et al. (2012) developed a positive pressure gripper that uses a balloon instead of a suction cup and can handle objects of various shapes [10]. Since this universal gripper can utilize both positive and negative pressure, it showed good performance in gripping objects of different size, shape, weight, and fragility. Reinforcement materials such as magnetorheological elastomers have also been utilized in suction cups of vacuum gripper systems to increase their performance [19]. As can be deduced from the above, vacuum grippers with one or more suction cups are frequently used to handle different types of objects. For example, Nakamoto et al. (2018) validated that a vacuum gripper can handle objects of 40 different shapes, sizes, and materials without requiring any changes in the gripper system setup [20].

As above mentioned, several studies have focused on how to effectively use a vacuum gripper and how to prevent a faulty gripper operation in advance. Operational parameters that affect a manufacturing process are usually categorized into machine parameters and product parameters. Whereas product parameters pertain to the production of the handled object in the process (such as the three-dimensional lengths of the object), machine parameters are related to the motion control of the installed actuators (for example, the speed of actuator movement). In general, for the effective execution of manufacturing processes, appropriate machine parameters are usually analyzed, instead of product parameters, because product parameters are already optimized before being fed into the manufacturing system. For example, Wang et al. reported that the type of pipe material and operating temperature can determine the suction power during gripper operation [21]. The authors [21] also investigated the effects of cup size and length of suction time on the gripping operation. According to another study [22], the performance of the gripping operation can depend on the shape of suction cups (universal, flat, or deep). In addition, 
considerable research has been performed on the effects of machine parameters, including suction cup size [23,24], number of cups [25], and initial applied pressure, on the suction operation [26].

However, these investigated machine parameters are usually "design variables" that are determined in the design phase of the product life cycle; thus, they cannot be easily modified later during production. In other words, it is difficult to adjust these parameters during each gripping operation in real time. Therefore, many studies have alternatively focused on approaches to detect defects in objects, such as dents or ripped sections on the objects, before the gripping operation because the prior detection of defects enables an error-free operation. For box-lifting operations, ultrasonic sensor signals have been utilized to identify the relative curvature of box surfaces [27]. Similarly, convolutional neural network models based on sensor signals have been proposed to classify normal and defective box surfaces [27]. Moreover, surface cleanliness has also been found to be important in the effective use of grippers [28]. The authors determined that the suction performance when using vacuum grippers can be determined by the number and size of contaminants on target surfaces. Since the condition of objects being handled cannot always be changed during production operations, the only feasible solution is to remove the defective objects in advance. In other words, it is still difficult to adjust certain conditions or parameters for recovering a fault state.

Therefore, this paper proposes a real-time predictive process adjustment method for pick-up operations using vacuum grippers. To this end, a pick-up operations testbed equipped with a two-way data monitoring and process parameter control module was developed. To analyze sensor signals in terms of faulty operation detection, a sensing module was devised by installing an air pressure sensor inside the Venturi line of a vacuum gripper, and the significant features that can help distinguish between normal and faulty gripper operation before the completion of the current pick-up operation or start of the next operation were characterized. Finally, an experiment to determine whether it is possible to recover faulty states by adjusting relevant process parameter(s) was performed.

The remainder of this paper is organized as follows: In Section 2, the developed system configuration to acquire appropriate sensor signals and provide feedback commands to the controller is explained in terms of hardware and software. In addition, air pressure sensor signals to obtain meaningful features that can help detect faulty operations in advance are analyzed. The experimental results are summarized in Section 3. The concept of predictive process adjustment in the pick-up operation of a vacuum gripper by controlling a related control parameter is also demonstrated. Lastly, in Section 4, the study is summarized and the scope for future work discussed.

\subsection{Literature Review on Fault Detection Methods}

Fault detection and diagnosis (FDD) approaches based on traditional statistical process control charts as well as advanced data mining techniques have been employed in various manufacturing applications [5,29-32]. As summarized in Table 1, statistical chart-based FDD approaches provide a satisfactory detection performance when sensor signals are only collected during the normal operation of a system, and the corresponding measurements are concentrated in one or several clusters. Principal component analysis (PCA) is a popular example of a fault diagnosis method based on traditional statistical process control charts where multivariate analog sensor signals are simultaneously collected from a manufacturing process [33-35]. This method reduces the dimensionality of original historical data according to eigenvectors decomposed via singular value decomposition [33]. Kim et al. (2020) employed PCA to derive a residual control chart to monitor the current system status [36]. Since highly correlated and dimensional sensor signals were fed as input data, the authors were able to extract hidden but meaningful features by combining traditional PCA with functional PCA. Since traditional PCA deals with linear relationships in the given multivariate dataset and functional PCA contains non-linear eigenfunctions and can 
handle highly correlated multivariate sensor signals, the derived features were used as indicators of process control charts; this yielded a superior fault detection performance.

Table 1. Classification of fault detection and diagnosis (FDD) approaches.

\begin{tabular}{|c|c|c|}
\hline & Statistical Chart & Data Mining \\
\hline Usage of fault state data & Unsupervised learning & Supervised learning \\
\hline Mathematical model & Yes & No \\
\hline $\begin{array}{c}\text { Gradient relationship between } \\
\text { measurements and } \\
\text { system operation states }\end{array}$ & Yes & Not necessary \\
\hline Popular decision criteria & $\begin{array}{l}\text { Distance from } \\
\text { the normal states }\end{array}$ & $\begin{array}{l}\text { Similarity to the known signal } \\
\text { behaviors during fault states }\end{array}$ \\
\hline
\end{tabular}

By contrast, data-mining-based FDD approaches are usually utilized when the sensor signals are excessively scattered regardless of the system operation state (normal or fault). Due to this characteristic, it is not easy to develop a robust representative mathematical model for quantifying the state of the system. That is, advanced machine learning techniques have been frequently applied when the collected sensor signals do not have distinguishable features between the normal and faulty system states [37]. In order to detect unknown faulty statuses more accurately, pattern classification with discrete state vectors (DSVs) was improved based on a similarity analysis [38]. Since previous DSV-based fault detection methods showed good performance when every DSV is discernible based on training data, the authors used Naïve Bayes approximation and the Brier score to evaluate unknown DSVs in terms of fault detection power. Based on the proposed technique, the faulty operation of a vehicle engine could be detected more precisely.

As the above approaches explained, most O\&M studies have focused on offline fault diagnosis $[5,35,36]$. Online fault detection is usually performed by comparing the current operation state and the offline fault detection model, and consequently, it gives the user alarm when the current state is considered as a fault state (or, a fault state will occur in near future. For effective manufacturing operations, corrective recovery action(s) should be manually performed by operators to prevent the detected faults. For example, for the effective operation of an automated storage and retrieval system (ASRS), Internetof-things-based controllers and sensors were installed [31]. Depending on the current system status determined by analyzing real-time vibration signals, the controller changes the relevant process parameter (i.e., motor speed in transporters) and, consequently, the ASRS can automatically maintain a failure-free status. In this regard, O\&M approaches have evolved from corrective maintenance, through preventive maintenance and conditionbased monitoring, to predictive maintenance [6,39]; however, it is still not easy to combine real-time fault detection/prediction and automatic process adjustment.

\section{Materials and Methods}

\subsection{Materials: System Configuration for Monitoring Pick-Up Operations in Real Time}

To identify the key machine parameters and test the predictive process adjustment with the corresponding characteristics, a testbed that performs a pick-up operation using a vacuum gripper [27], as depicted in Figure 2 was constructed. Furthermore, the monitoring and control modules were upgraded, as shown in Figure 2b. The detailed information of the system configuration is summarized in Table 2. 


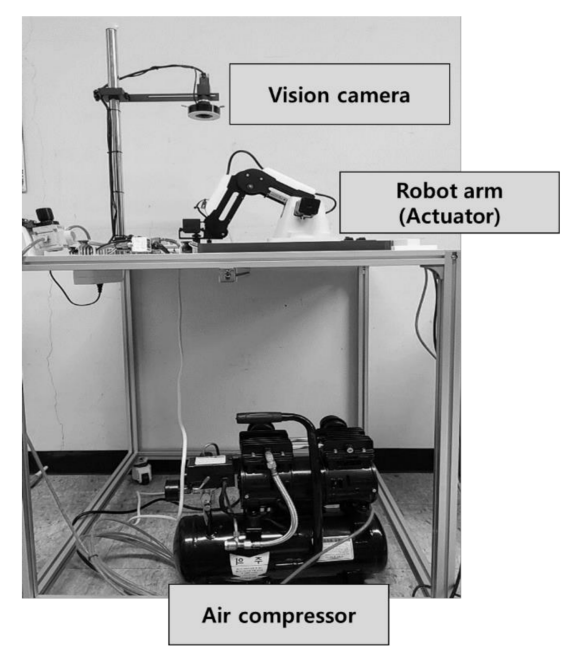

(a)

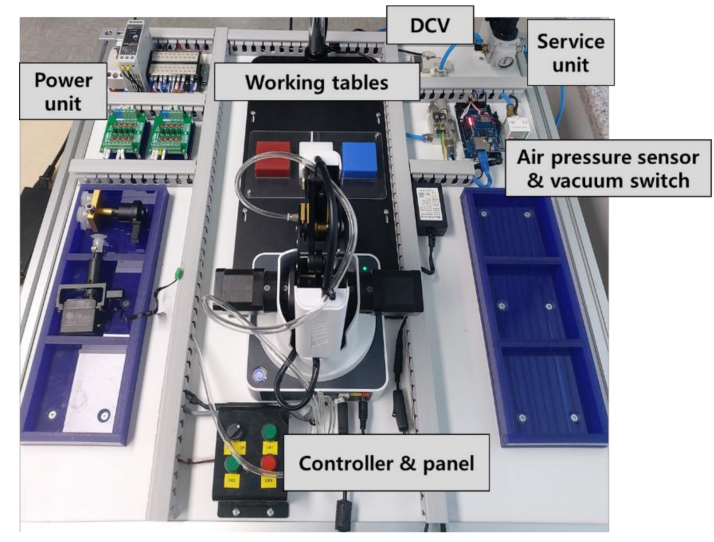

(b)

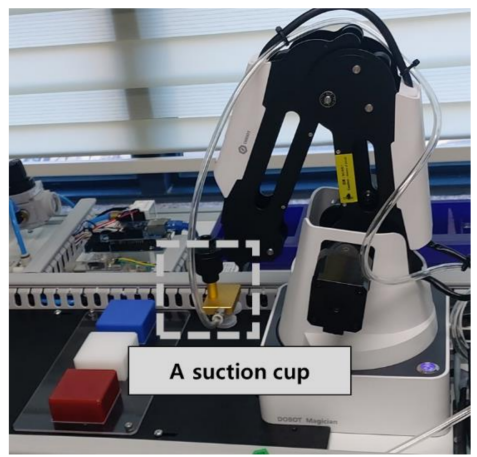

(c)

Figure 2. Testbed for performing the pick-up operation and monitoring the amount of applied air pressure during each operation cycle: (a) front view; (b) top view; (c) closed view for a suction cup.

Table 2. Summary of testbed configuration.

\begin{tabular}{|c|c|}
\hline Unit & Function \\
\hline Air compressor & Supply compressed air \\
\hline Air pressure sensor & Measure air pressure during operation \\
\hline Air pressure gauge & Measure an initially applied amount of air pressure \\
\hline Vacuum switch & Detect success / failure of suction \\
\hline Four-axis robot arm & Move vacuum gripper \\
\hline $\begin{array}{l}\text { Vacuum gripper with a suction cup } \\
\text { and single solenoid } \\
\text { directional control valve (DCV) }\end{array}$ & Conduct suction operation \\
\hline Arduino-based controller & Collect signals and send them to the PC \\
\hline PC-based controller & Control the robot arm and Arduino-based controller \\
\hline
\end{tabular}

The outlet air pressure was considered as an indicator to identify the current pick-up operation (i.e., suction operation) in real time, instead of the inlet air pressure, which is most commonly measured. Among various machine parameters, the outlet air pressure is the most appropriate variable because it changes depending on whether the suction is turned on or not. In a vacuum gripper, compressed air is supplied from an air compressor to the suction cup through a Venturi line. The Venturi line is designed to increase airflow speed by changing the size of the air pipe [40]. When the compressed air moves from the entrance to the exit of a Venturi line, a partial vacuum state occurs at the jet nozzle (an additional narrow vertical air pipe between the entrance and exit of the Venturi line). Regardless of 
whether a partial vacuum state was created, it was assumed that the magnitude of the outlet air pressure changed.

For predictive process adjustment according to real-time operation monitoring, the control modules and the corresponding control software were upgraded, as shown in Figure 3. Due to the upgrade, it was not only possible to acquire sensor signals in real time but also to simultaneously change machine parameters, such as the $\mathrm{x}-, \mathrm{y}-$, and $\mathrm{z}$-positions corresponding to the suction start, during the operation. The workflow is as follows:

1. Make sure every module is turned on and connected to the PC-based controller via Ethernet communication.

2. Send a pick-up operation message to move the robot arm and start the suction operation.

3. When the suction is started by the PC-based controller, analog sensor signals (outlet air pressure) are simultaneously acquired in real time and can be monitored through the developed control software.

4. After performing the given pick-up operation (regardless of the operation success), the acquired sensor signals are exported as a spreadsheet per operation.

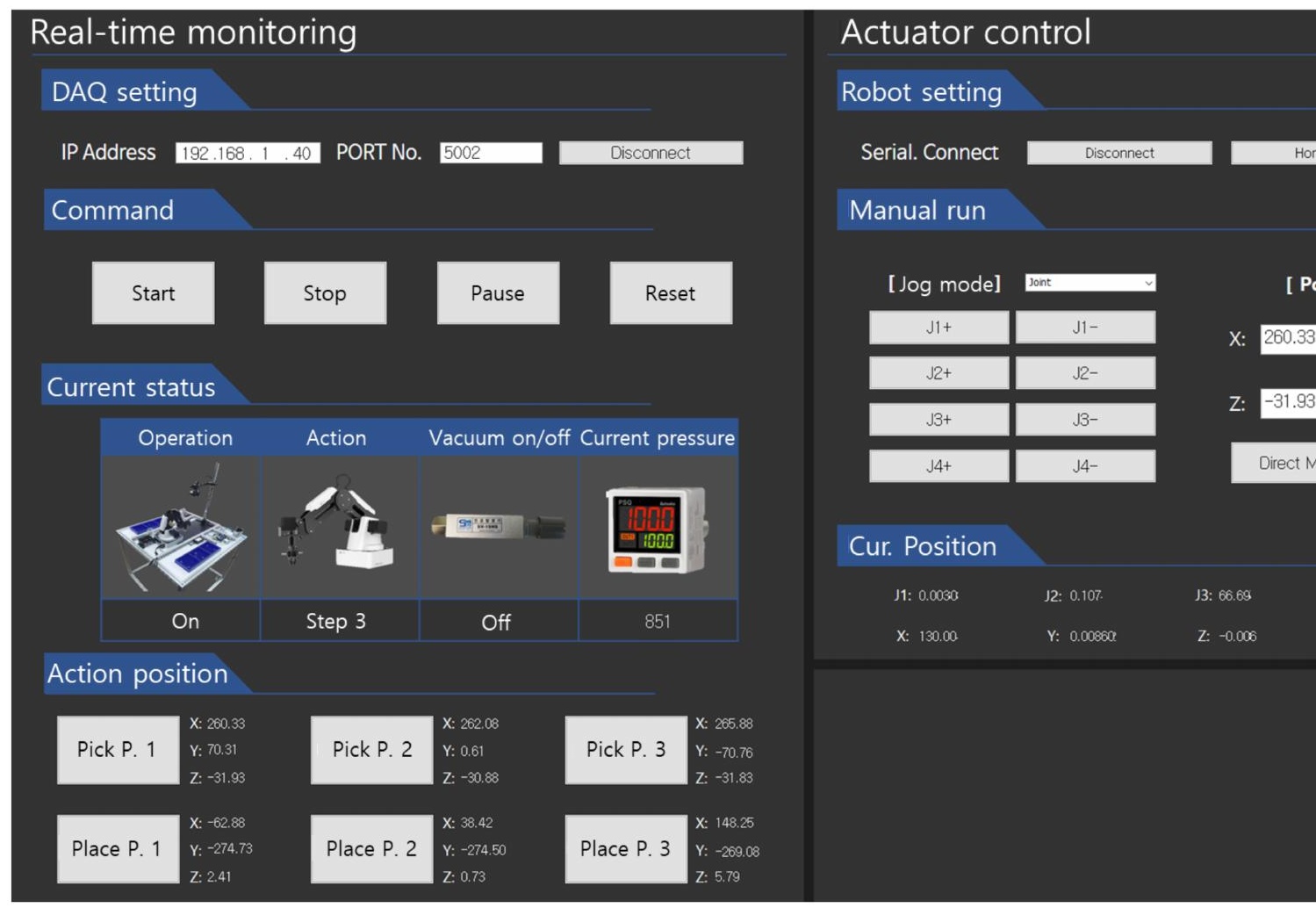

Figure 3. Software to control the testbed, collect sensor signals in real time, and send feedback control messages.

\subsection{Methods}

Predictive process adjustment is a representative approach in predictive maintenance. The concept of this approach is to automatically perform appropriate feedback actions, which are determined by detecting and diagnosing the current system status or predicting an upcoming fault status through real-time monitoring [31]. Therefore, in this paper, early fault detection and recovery actions are proposed in order to conduct predictive process adjustment in real time, as illustrated in Figure 4. 


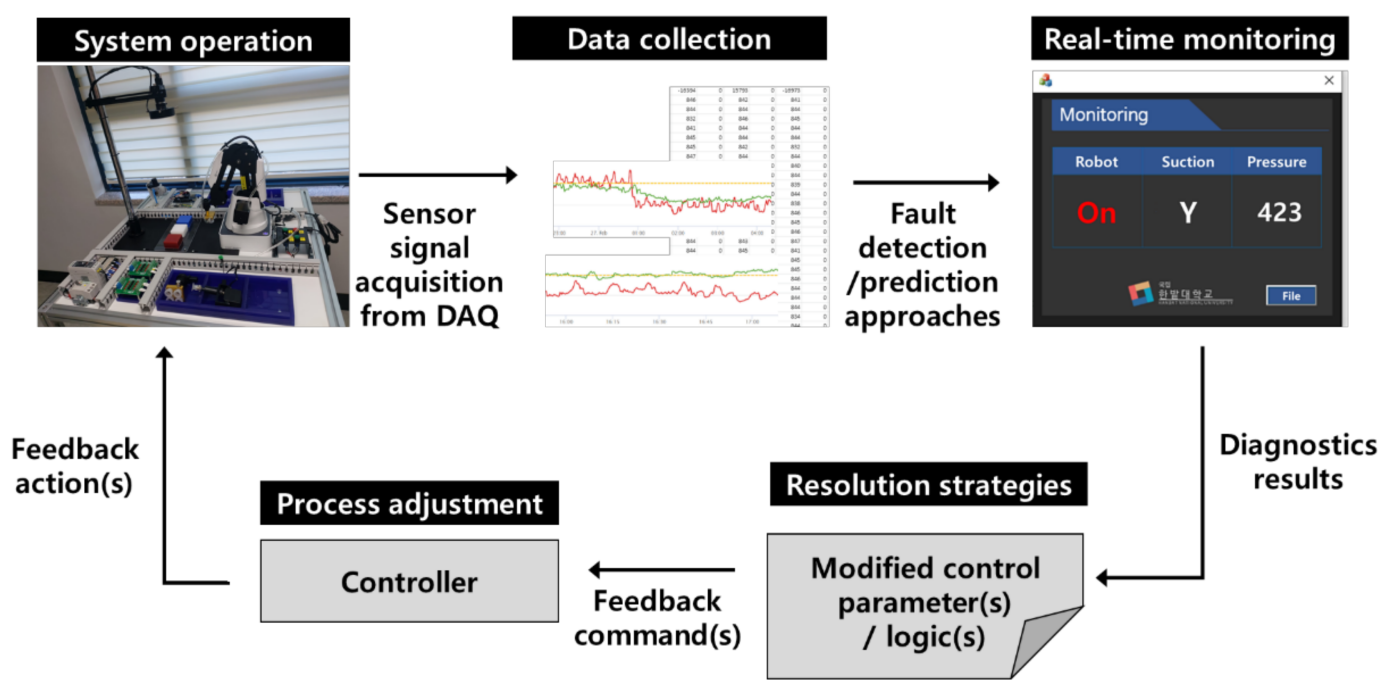

Figure 4. Framework of the predictive process adjustment method based on real-time data acquisition and feedback control.

First, the relevant measurements are acquired from the testbed when a vacuum gripper performs a pick-up operation according to the controller's command. During the training phase, the time-series sensor signals for every cycle are accumulated in a history dataset, which is employed to generate a fault detection model. To develop a fault detection model that can identify an unknown and sudden fault situation in advance, a detection threshold should be determined. Since sensor signals were already collected, it is possible to construct fault detection model using several fault detection/prediction approaches, from conventional statistical models to pattern extraction-based deep learning models. In this study, the simplest statistical-based fault detection model was employed using Equation (1) [41]:

$$
\text { Detection threshold }=\overline{\bar{X}}+\frac{3}{d_{2} \sqrt{n}} \times(\bar{R}),
$$

where $\overline{\bar{X}}$ is (approximately) the mean of the sensor signals a short duration after the suction command is given to an actuator in normal operation; $\bar{R}$ is the mean of the differences of maximum and minimum measurements in the same dataset, which is used for calculating $\overline{\bar{X}}$; and $d_{2}$ is a constant value chosen by the number of sensor measurements (i.e., $n$ ).

For a statistical process control approach, the detection threshold is computed according to the $\bar{X}$-chart. The $\bar{X}$-chart is one of the control charts that is used for identifying whether measurements in the current batch are under the statistical control or not. It can be categorized as unsupervised learning, as history datasets collected during normal operations are used to develop a detection model, such as those based on $\overline{\bar{X}}$ and $\bar{R}$ computation. The $\bar{X}$-chart was utilized in this study because the time-series sensor signals collected during one pick-operation operation are considered as a dataset in one batch. In this regard, a series of sensor signals is collected for a short duration after the suction starts (during one operation cycle) in order to assume that the collected measurements are under identical conditions.

In common process control chart models [41], the upper and lower control limits are applied together to determine the statistical outliers. However, in this study, the direction of the failure status was clearly determined (e.g., a higher value indicates a failure of vacuum generation, and hence, an operation failure); therefore, only the upper control limit was used as the detection threshold. Using the derived detection threshold, the failure status of the pick-up operation was detected when the following condition is not satisfied:

$$
x_{i} \leq \alpha \times \text { Detection threshold, }
$$


where $x_{i}$ is the current sensor value and $\alpha$ is the predefined confidence interval.

If a fault is detected during real-time monitoring based on the above detection model, the appropriate resolution strategy is consequently developed. In this study, the machine parameter was carefully controlled in order to overcome situations with faults.

\section{Experimental Results}

\subsection{Result: Early Fault Detection for Pick-Up Operation}

Using the testbed described in Section 2, a pick-up experiment was conducted to determine the characteristics of the collected sensor signals depending on the success or failure of the suction operation. The target operation was to lift rectangular boxes using one suction cup. To generate two different states (i.e., success and failure to lift), the following two different types of boxes were used, as shown in Figure 5:

- Box type I: Normal box with a surface that is sufficiently flat to be picked up with conventional vacuum grippers and judged to be acceptable based on product quality inspection.

- Box type II: Box that appears normal in the product quality inspection but is not flat enough to be picked up using a conventional vacuum gripper ("box type $\mathrm{II}^{\prime}$ hereinafter) owing to a slight concave curvature in its contact surface, resulting in a faulty pick-up operation.

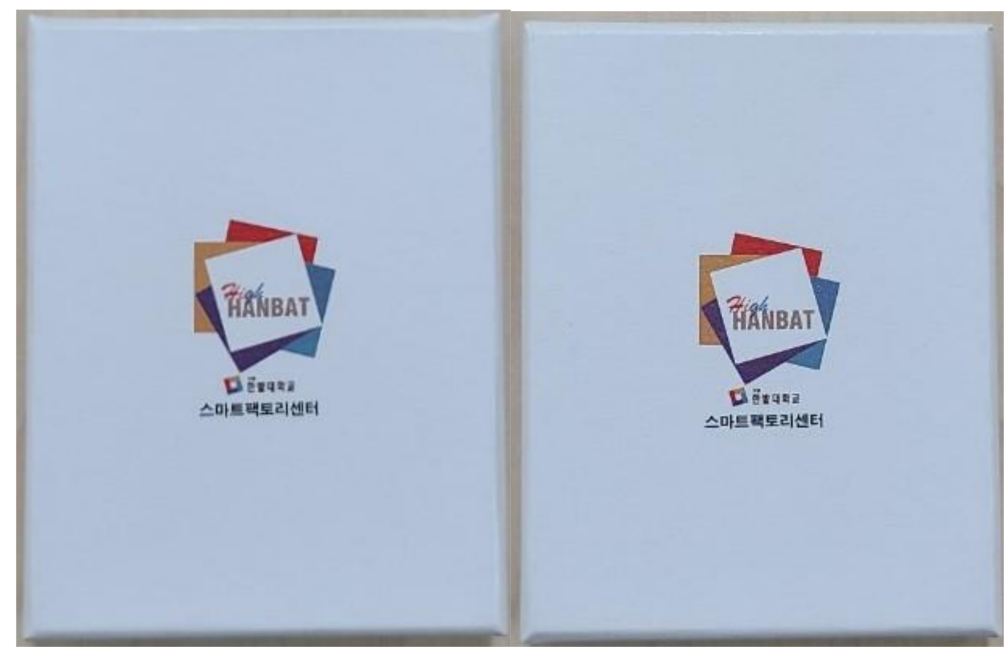

(a)

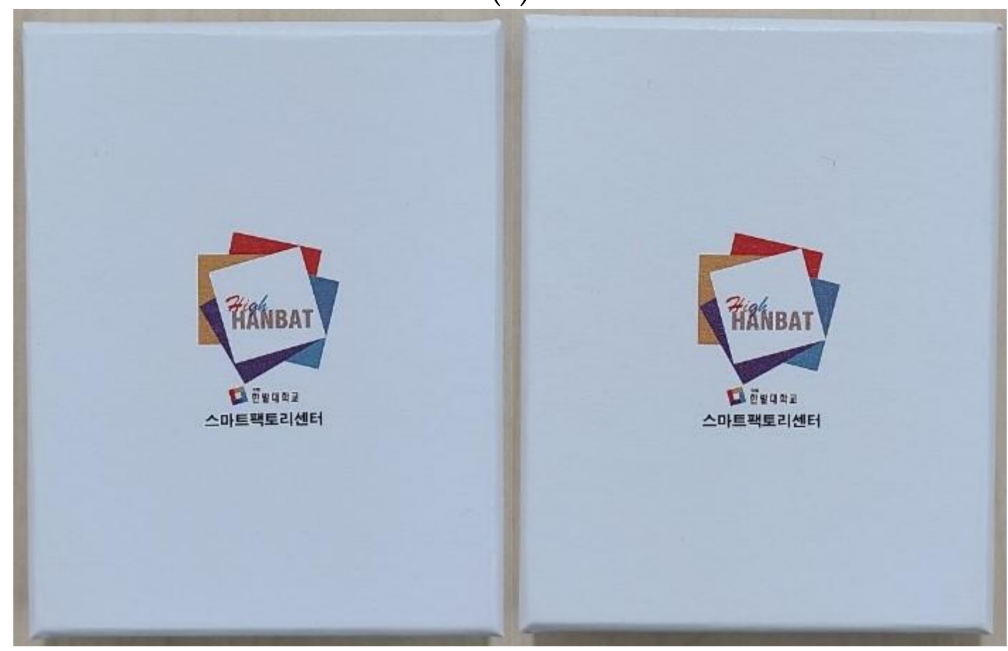

(b)

Figure 5. Target objects: (a) two samples of box type I; (b) two samples of box type II. (They do not show any significant difference in 2D images). 
Both types of box cover, box type I and II, are considered as normal quality in product quality inspection phase. Although box type II should be used in the manufacturing process from the viewpoint of product quality, because of its very negligible concave on the contact surface, it results in a faulty pick-up operation. Importantly, even if the manufacturer attempts to install a system to remove box type II from the production line, it is highly difficult to distinguish between the two types using the naked eye or a 2D camera, as depicted in Figure 5.

The lifting operation was conducted 20 times for each box type, and the corresponding sensor signals were collected, as illustrated in Figure 6. The generated historical data were recorded with the corresponding timestamps. The duration was approximately $10 \mathrm{~s}$, and the analog signals were recorded at a sampling rate of approximately $10 \mathrm{~Hz}$. In addition, missing or noisy data in the collected raw signals were filtered using adjacent measurements.

Figure 6a describes the pressure signals of the airflow in the Venturi channel for every lifting iteration. Before starting the suction operation using the vacuum gripper, it was ensured that the initial pressure of the airflow in the Venturi line was almost identical to the original air pressure provided by the air service unit and air compressor. After starting the suction operation (before lifting the object), it can be observed in Figure 6a that the monitored air pressure decreased continuously (at approximately 5.6 to $6.0 \mathrm{~s}$ ). When the suction was sufficiently succeeded, the decreased pressure was constantly maintained during the rest of the pick-up operation, until moving to the destination position, and the decreased level was identical in the 20 repetitions. Although certain fluctuations in the inlet pressure can be observed during the pick-up operation, the decrease in pressure was mostly constant until the suction operation was terminated. Signal collection automatically stopped after the robotic arm was moved with the vacuum gripper to the destination position and the vacuum switch was turned off to release the object.

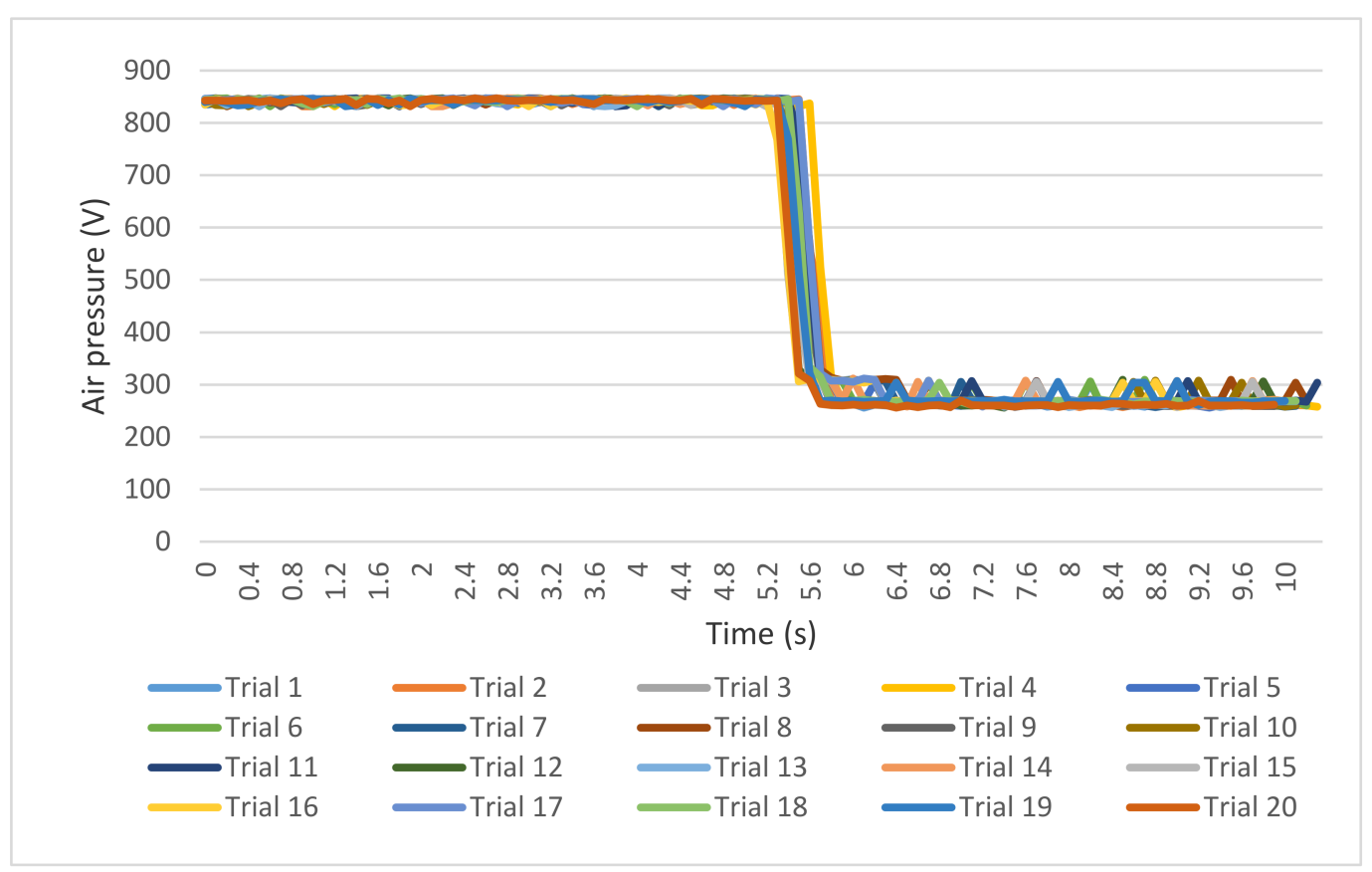

Figure 6. Cont. 


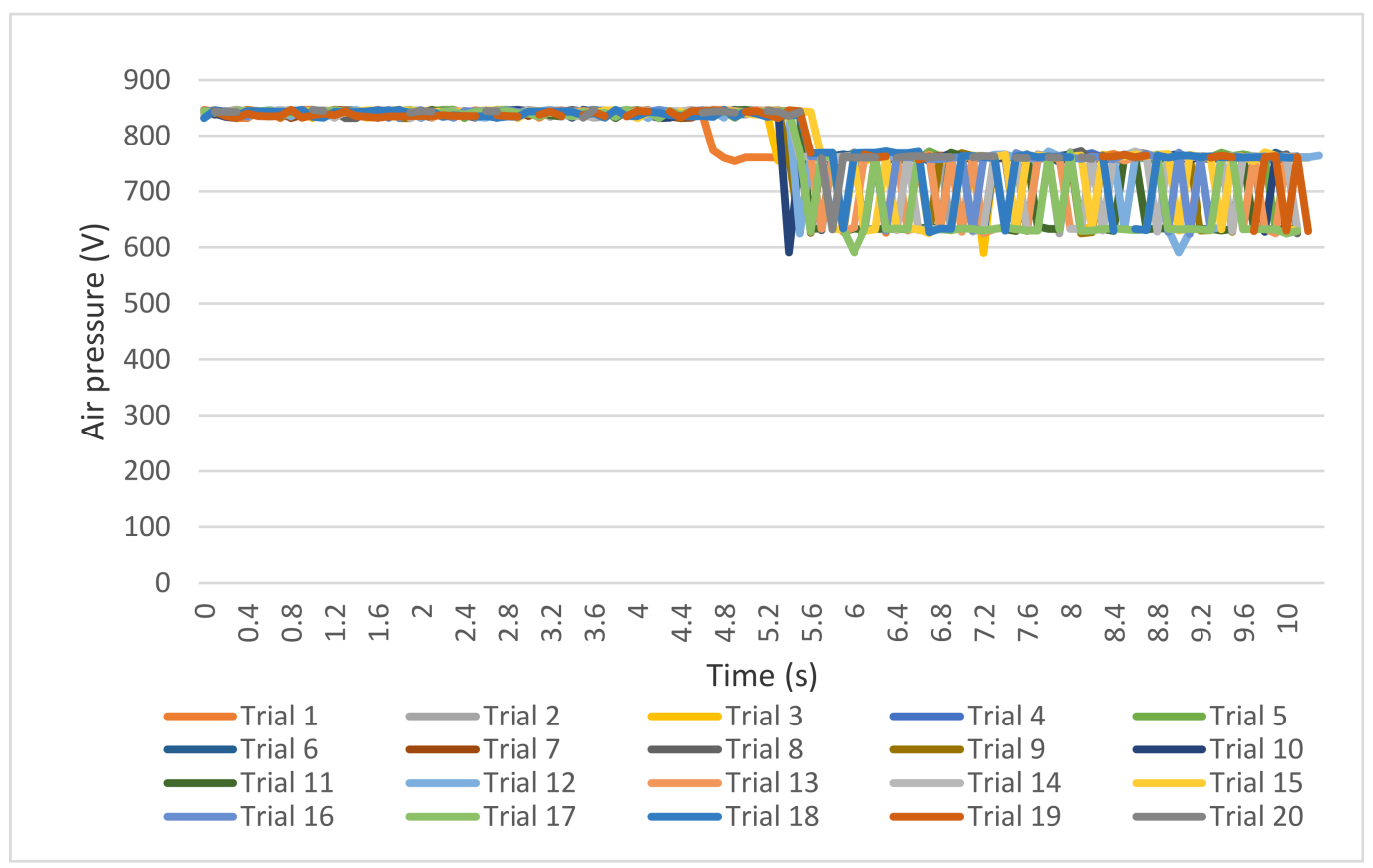

(b)

Figure 6. Measured air pressure over time: (a) successful lift for box type I; (b) failed lift for box type II.

For box type II, the gripper failed to pick-up the box in all repetitions, as listed in Table 3, even though the conditions were kept identical to those for box type I. As depicted in Figure $6 \mathrm{~b}$, the air pressure starts to decrease when the suction operation starts, but the level of decrease is different from that for box type I. As a result of the negligible concave curvature on the contact surface, it is not possible to generate sufficient suction, and the amount of outlet air pressure cannot decrease sufficiently for a successful gripper operation. In Figure 6b, the sensor signal for Trial 1 is slightly different from those for the other 19 trials; this is due to an inadvertent early manual control suction start command by the operator.

Table 3. Pick-up operation results.

\begin{tabular}{cc}
\hline Box Types & Operation Success Rate \\
\hline Box type I & $100 \%$ \\
Box type II & $0 \%$ \\
\hline
\end{tabular}

In conventional operations with a vacuum gripper, a vacuum switch determines whether the suction operation was successful after the pick-up operation has been conducted. In other words, although the vacuum switch does not indicate that a sufficient vacuum has been successfully generated, the gripper (and the connected robotic arm) moves to the next position regardless of the completion of the current pick-up operation. However, upon using the information derived from this study, extraneous movements or operations without the target object can be prevented.

In summary, the process can be summarized as follows:

1. Start the suction operation and measure the amount of outlet air pressure in real time.

2. Check the decrease during approximately short time period (i.e., 5.6 to $6.0 \mathrm{~s}$ ), which corresponds to suction start.

3.1. If there is a sufficient decrease in air pressure, then conduct the next step (usually, move the gripper to the next position). 
3.2. If the air pressure does not decrease to the desired level, then conduct the predictive process adjustment until the object is lifted by the gripper.

\subsection{Discussion: Conducting Appropriate Recovery Actions}

After predicting the outcome of a pick-up operation (as already described in Section 3.1), it is necessary to identify which control parameter(s) can result in a failed pick-up operation. There are several control parameters whose value changes during the gripper operation, such as the initial applied pressure, length of suction time, and suction position [26,27]. In this study, the vertical position of the vacuum gripper at the suction start (hereafter called as the "z-position") was selected as the control parameter for the predictive process adjustment for the pick-up operation. The z-position is illustrated in Figure 7: it represents the bottom position of the suction cup attached on a vacuum gripper. In the case of more than one suction cup, the same z-position is recommended for all suction cups.

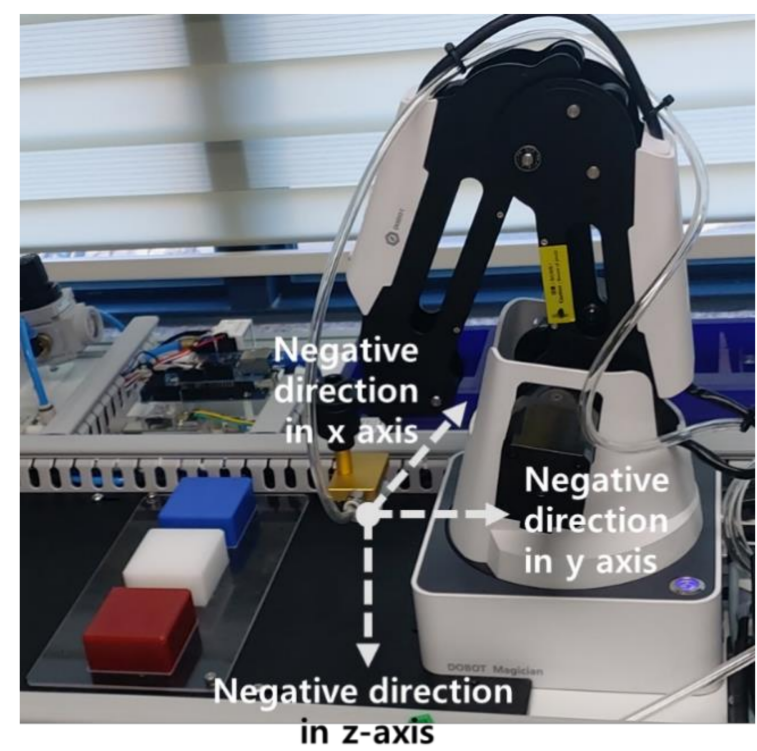

(a)

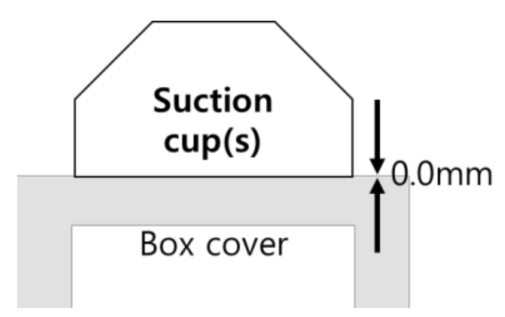

(b)

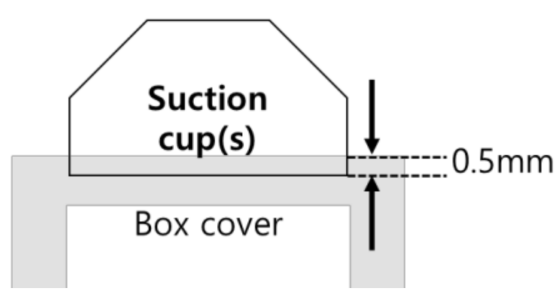

(c)

Figure 7. Suction cup positions for predictive process adjustment: (a) axis information for suction cup control; (b) diagram for the $0.0 \mathrm{~mm}$ z-position; (c) diagram for the $-0.5 \mathrm{~mm}$ z-position.

Experiments were conducted to investigate the effect of the z-position on the pick-up operation, as summarized in Table 4 . The pick-up operation consisted of the following consecutive steps: movement to the suction location, suction start, upward movement, movement to the destination, and suction finish. 
Table 4. Experimental setup to determine effect of z-position on the pick-up operation.

\begin{tabular}{cc}
\hline Dependent variable & $\begin{array}{c}\text { Amount of outlet air pressure } \\
\text { at approximately } 5.6 \text { to } 6.0 \mathrm{~s}\end{array}$ \\
Independent variable & $\begin{array}{c}\text { Z-position with four levels }(0.0,-1.0,-1.5, \\
\text { and }-0.0 \mathrm{~mm})\end{array}$ \\
Number of repetitions & 10 times for each condition \\
\hline
\end{tabular}

Once the command was sent to the vacuum gripper, the sensor signals were collected in the form of a time series from step 2 to step 3. A total of 40 time-series datasets were recorded. Using mean values corresponding to approximately 5.6 to $6.0 \mathrm{~s}$ for each timeseries dataset, a one-way analysis of variance (ANOVA) was conducted to determine the statistical effects of the dependent variable on the independent variable. ANOVA is a typical statistical test to determine the source of measurement differences, that is, whether the differences result from variance between groups (levels) or from variance within groups corresponding to independent variables [42]. It can be treated as the extended version of the t-test for more than three groups corresponding to an independent variable. This statistical test is prevalently employed to determine the effects of controllable parameters on the output [43-45].

The results are summarized in Table 5, where the z-position shows a significant difference in the pick-up operation ( $p$-value $<0.05)$. As can be observed from Table 6 , the success ratio of the operation increased from $0 \%$ to $100 \%$ as the vertical position at the suction start lowered. Specifically, the suction cup failed in nine trials except in the case of Trial $5(-1.0 \mathrm{~mm})$, whereas it failed in only three trials for box type II (i.e., Trials 1,2 , and 6 ) with the z-position at $-1.5 \mathrm{~mm}$. Finally, when the z-position was set as $-2.0 \mathrm{~mm}$, the gripper succeeded in picking the target up in every trial. Clearly, the outlet air pressure decreased by more (approximately 200 to $300 \mathrm{~V}$ ) than the decrease in the cases of operation failures (approximately 600 to $700 \mathrm{~V}$ ), and the outlet air pressure was continuously maintained until step 3, as depicted in Figure 8.

Table 5. ANOVA results of the effect of the z-position on the pick-up operation of the gripper.

\begin{tabular}{cccccc}
\hline Source & SS & df & MS & F & $p$-Value \\
\hline Between groups & $1,777,360$ & 1 & $1,777,360$ & 1284 & $<0.000$ \\
Within groups & 52,616 & 38 & 1385 & & \\
Total & $1,829,976$ & 39 & & & \\
\hline
\end{tabular}

*SS: sum of squares, df: degree of freedom, MS: mean squares.

Table 6. Experimental results of the predictive process adjustment by controlling the z-position of suction start with box type II.

\begin{tabular}{cc}
\hline Z-Position & Operation Success Rate \\
\hline $0.0 \mathrm{~mm}$ & $0 \%$ \\
$-1.0 \mathrm{~mm}$ & $10 \%$ \\
$-1.5 \mathrm{~mm}$ & $70 \%$ \\
$-2.0 \mathrm{~mm}$ & $100 \%$ \\
\hline
\end{tabular}




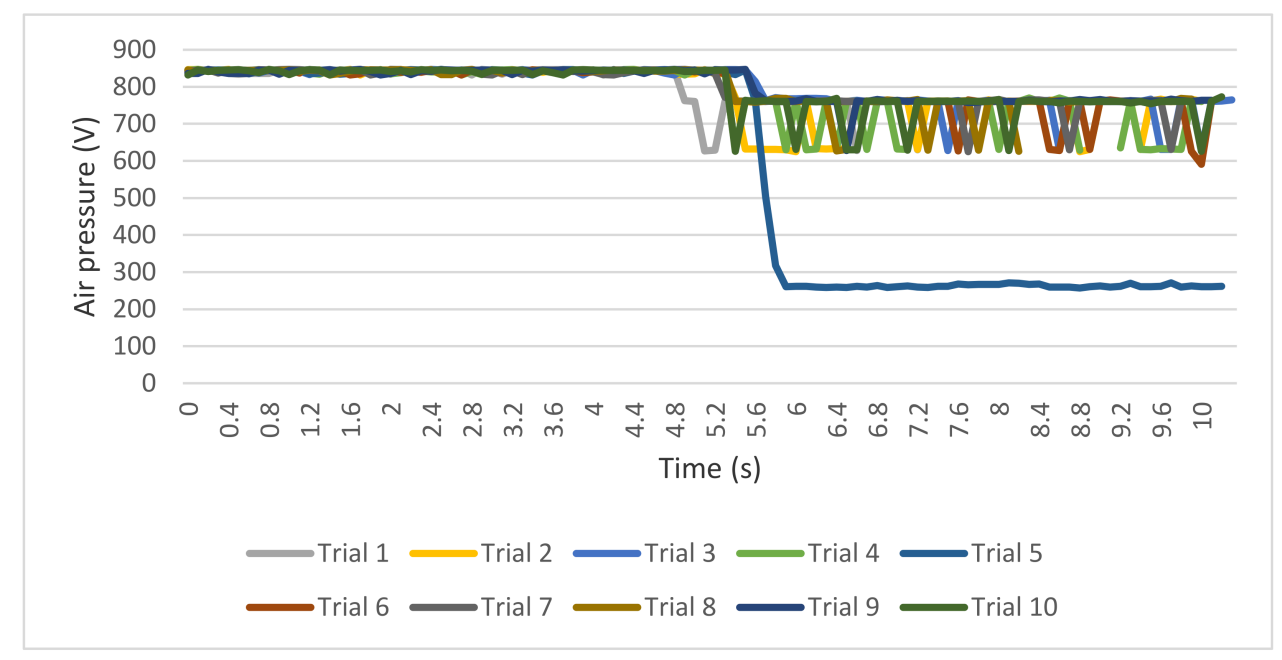

(a)

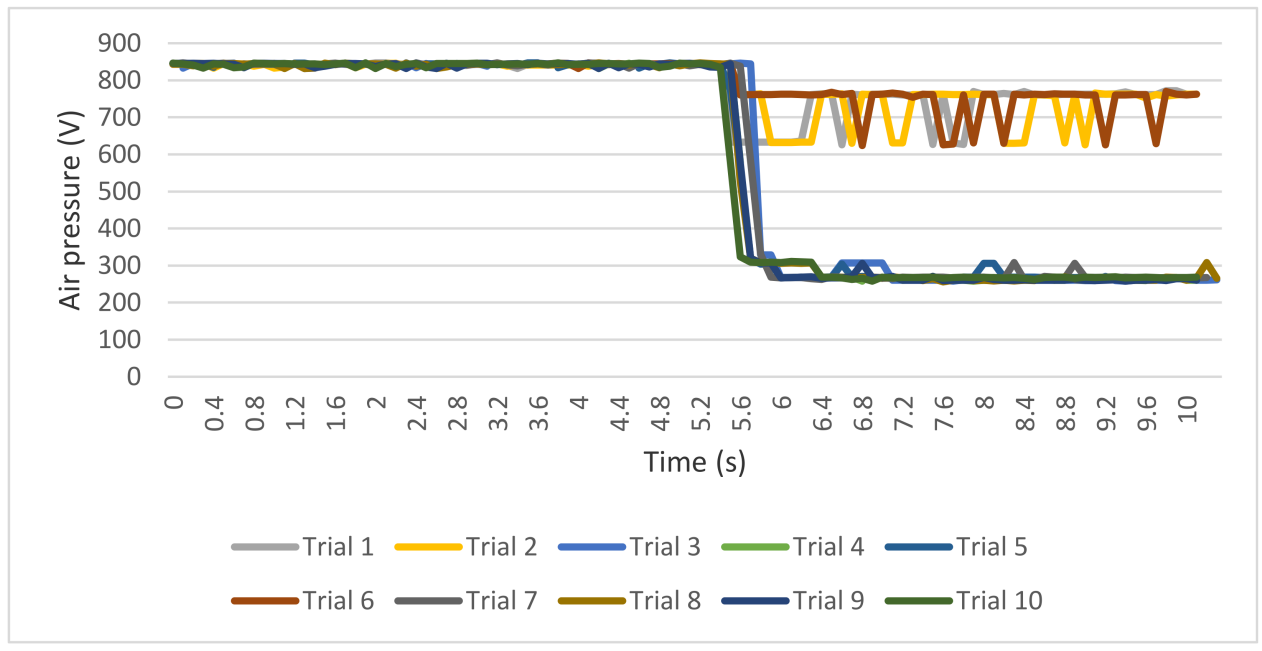

(b)

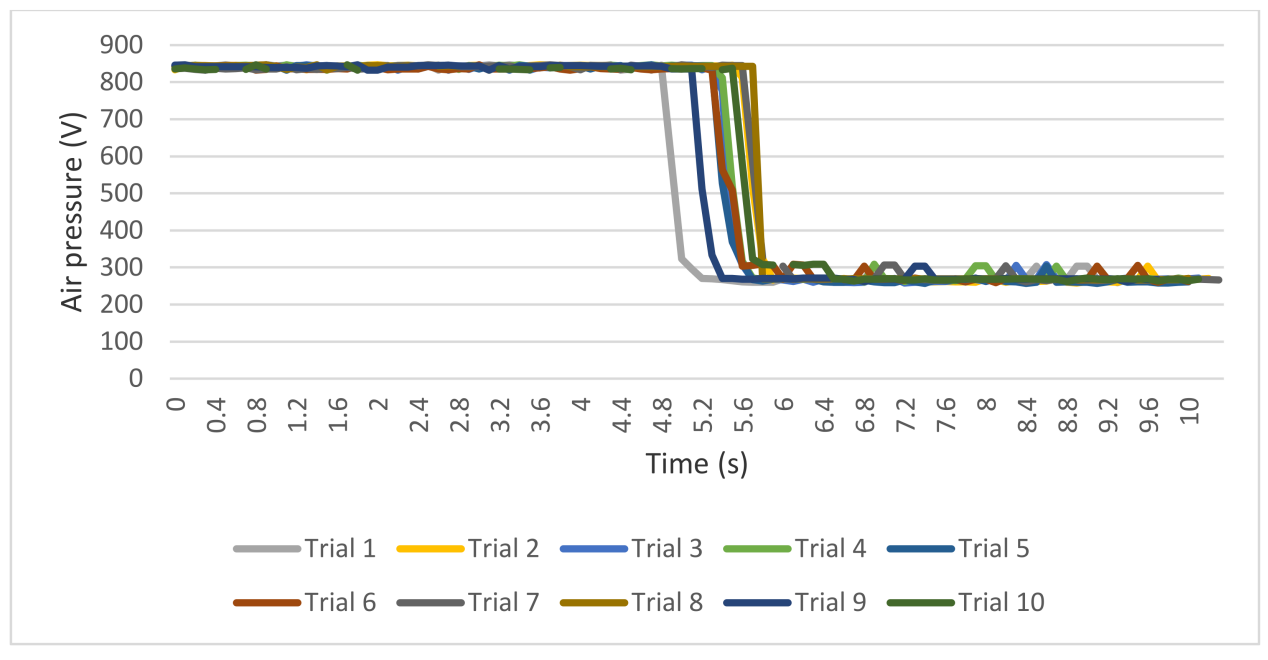

(c)

Figure 8. Collected air pressure signals depending on the z-position (during pick-up operation with box type II): z-position is set as (a) $-1 \mathrm{~mm}$; (b) $-1.5 \mathrm{~mm}$; and (c) $-2.0 \mathrm{~mm}$. 
In summary, the predictive process adjustment with real-time operation monitoring can be conducted as follows:

1. Start the suction operation and measure the amount of outlet air pressure in real time.

2. Check the decrease during approximately a short time period (i.e., 5.6 to $6.0 \mathrm{~s}$ ), which corresponds to suction start.

3.1. If the air pressure decreases sufficiently, then perform the next step (usually, move the gripper to the next position).

3.2. If a suction step is considered a failure (i.e., air pressure does not decrease to the predefined UCL), move the z-axis downward until the air pressure falls to the UCL.

However, as the z-position is further lowered, the probability of defects on the contact surface increases. A vacuum gripper that lowers excessively may result in a dent or a hole, and consequently, the object will be considered to be defective right before the pick-up operation. Therefore, it is essential to determine the appropriate z-position that does not cause any damage to the surface of the object while maximizing the success rate of the pick-up operation.

\section{Conclusions}

In this paper, a testbed for conducting a pick-up operation using a vacuum gripper with a single suction cup was prepared. Using the proposed method, the air pressure in the Venturi line was automatically monitored in real time. When a command for starting suction was provided to the gripper, a sharp decrease in the collected air pressure signals appeared at approximately $0.5 \mathrm{~s}$. However, the same decrease was not observed in the signal for faulty box surfaces; consequently, the suction action and the corresponding gripper operation were not performed owing to insufficient contact between suction cup(s) and the contact surface of the object. Using the early detection results derived from the air pressure signal analysis, a prediction-based process adjustment method for the pick-up operation was proposed. Through pick-up experiments using the developed testbed, it was revealed that the z-position of the suction cup significantly affects whether an object is properly gripped by the vacuum gripper or not. Therefore, it is possible to determine a possible error situation in advance (before failure of the lifting operation) and provide appropriate feedback control commands so that the target operation is finished successfully without stopping machine operations.

However, for stable operation and generalization, it is necessary to conduct further research on the following: (i) identifying the appropriate depth for the z-position that does not generate any defects on a contact surface but maximizes the rate of success of the pick-up operation; (ii) generalizing the results for different materials, sizes, weights, or shapes of handled objects and various configurations of vacuum grippers; and (iii) combining the machine-status monitoring result and product defect detection result to improve the productivity and product quality of an industrial production system.

Author Contributions: Conceptualization, methodology, validation, formal analysis, investigation, resources, data curation, writing, visualization, supervision, project administration, funding acquisition, S.B.; Experiment, D.O.K. All authors have read and agreed to the published version of the manuscript.

Funding: This work was supported by the National Research Foundation of Korea (NRF) grant funded by the Korea government (MSIT) (No. NRF-2019R1G1A1097478) and was also supported by Korea Institute for Advancement of Technology (KIAT) grant funded by the Korea Government (MOTIE) (P0012744, The Competency Development Program for Industry Specialist). In addition, this research was supported by the research fund of Hanbat National University in 2020.

Data Availability Statement: Not applicable.

Acknowledgments: The staffs of GL Company (Republic of Korea) provided immense support in building the experimental system. 
Conflicts of Interest: The authors declare no conflict of interest. The funders had no role in the design of the study; in the collection, analyses, or interpretation of data; in the writing of the manuscript; or in the decision to publish the results.

\section{References}

1. Bai, S.; Cheng, Z.; Guo, B. Maintenance optimization model with sequential inspection based on real-time reliability evaluation for long-term storage systems. Processes 2019, 7, 481. [CrossRef]

2. Rahman, M.; Zaccaria, V.; Zhao, X.; Kyprianidis, K. Diagnostics-oriented modelling of micro gas turbines for fleet monitoring and maintenance optimization. Processes 2018, 6, 216. [CrossRef]

3. Lin, M.; Ruigang, T.; Yan, R.; Lei, Z.; Xizhen, S. Research on identification and measurement methods of influencing factors of investment in operation and maintenance. In E3S Web Conference 236, 3rd International Conference on Energy Resources and Sustainable Development (ICERSD 2020); 2021; p. 04010. Available online: https://www.e3s-conferences.org/articles/e3sconf/ abs/2021/12/e3sconf_icersd2020_04010/e3sconf_icersd2020_04010.html (accessed on 9 February 2021).

4. Furumoto, K.; Kolehmainen, A.; Silverajan, B.; Takahashi, T.; Inoue, D.; Nakao, K. Toward automated smart ships: Designing effective cyber risk management. In Proceedings of the 2020 International Conferences on Internet of Things and IEEE Green Computing and Communications and IEEE Cyber, Physical and Social Computing and IEEE Smart Data and IEEE Congress on Cybermatics, Rhodes, Greece, 2-6 November 2020; pp. 100-105. [CrossRef]

5. Espadinha-Cruz, P.; Godina, R.; Rodrigues, E.M.G. A review of data mining applications in semiconductor manufacturing. Processes 2021, 9, 305. [CrossRef]

6. Kim, D.-Y.; Park, J.W.; Baek, S.; Park, K.-B.; Kim, H.-R.; Park, J.-I.; Kim, H.-S.; Kim, B.-B.; Oh, H.-Y.; Namgung, K.; et al. A modular factory testbed for the rapid reconfiguration of manufacturing systems. J. Intell. Manuf. 2020, 31, 661-680. [CrossRef]

7. Townsend, W. The BarrettHand Grasper-Programmably flexible part handling and assembly. Ind. Robot Int. J. 2000, 27, 181-188. [CrossRef]

8. Li, C.; Gu, X.; Ren, H. A cable-driven flexible robotic grasper with Lego-like modular and reconfigurable joints. IEEE/ASME Trans. Mechatron 2017, 22, 2757-2767. [CrossRef]

9. Shao, G.; Ware, H.O.T.; Huang, J.; Hai, R.; Li, L.; Sun, C. 3D printed magnetically-actuating micro-gripper operates in air and water. Addit. Manuf. 2021, 38, 101834

10. Amend, R.; Brown, E.; Rodenberg, N.; Jaeger, H.M.; Lipson, H. A positive pressure universal gripper based on the jamming of granular material. IEEE. Trans. Rob. 2012, 28, 341-350. [CrossRef]

11. Vedhagiri, P.J.G.; Prituja, A.V.; Li, C.; Zhu, G.; Thakor, N.V.; Ren, H. Pinch grasp and suction for delicate object manipulations using modular anthropomorphic robotic gripper with soft layer enhancements. Robotics 2019, 8, 67. [CrossRef]

12. Chen, T.; Wang, Y.; Yang, Z.; Liu, H.; Liu, J.; Sun, L. A PZT actuated triple-finger gripper for multi-target micromanipulation. Micromach 2017, 8, 33. [CrossRef]

13. Matsuo, I.; Shimizu, T.; Nakai, Y.; Kakimoto, M.; Sawasaki, Y.; Mori, Y.; Sugano, T.; Ikemoto, S.; Miyamoto, T. Q-bot: Heavy object carriage robot for in-house logistics based on universal vacuum gripper. Adv. Robot. 2020, 34, 173-188. [CrossRef]

14. Jamaludin, A.S.; Razali, M.N.M.; Jasman, N.; Ghafar, A.N.A.; Hadi, M.A. Design of spline surface vacuum gripper for pick and place robotic arms. J. Mod. Manuf. Syst. Technol. 2020, 4, 48-55. [CrossRef]

15. Ashwini, K.; Rudraswamy, S.B. Automated inspection system for automobile bearing seals. Mater. Today Proc. 2020. [CrossRef]

16. Seo, J.W.; Lee, J.K.; Lee, S.; Lee, K.C. Slip detection of robot gripper with flexible tactile sensor. J. Korean Soc. Precis. Eng. 2014, 31, 157-164. [CrossRef]

17. Lee, J.K.; Kim, H.J.; Lee, S.; Lee, K.C. Design of grasping objects system of robot gripper using artificial neural network. In Proceedings of the 2014 KSMPE Conference, Changwon, Korea, 6-8 November 2014; p. 109.

18. Jaiswal, A.K.; Kumar, B. Vacuum gripper-An important material handling tool. Int. J. Sci. Technol. 2017, 7, 1-8.

19. Zhang, P.; Kamezaki, M.; Otsuki, K.; He, Z.; Sakamoto, H.; Sugano, S. Development of a vacuum suction cup by applying magnetorheological elastomers for objects with flat surfaces. In Proceedings of the 2020 IEEE/ASME International Conference on Advanced Intelligent Mechatronics (AIM), Boston, MA, USA, 6-9 July 2020; pp. 777-782.

20. Nakamoto, H.; Ohtake, M.; Komoda, K.; Sugahara, A.; Ogawa, A. A gripper for robustly picking various objects placed densely by suction and pinching. In Proceedings of the 2018 IEEE/RSJ International Conference on Intelligent Robots and Systems (IROS), Madrid, Spain, 1-5 October 2018; pp. 6093-6098.

21. Wang, J.; Liang, Y.; Zheng, Y.; Gao, R.X.; Zhang, F. An integrated fault diagnosis and prognosis approach for predictive maintenance of wind turbine bearing with limited samples. Renew. Energy 2020, 145, 642-650. [CrossRef]

22. Jaiswal, A.K.; Kumar, B. Design constraints of vacuum cup gripper of robots-as a pick and place operation tool. Int. J. Sci. Technol. 2016, 6, 26-35.

23. Jaiswal, A.K.; Kumar, B. Vacuum cup grippers for material handling in industry. Int. J. Innov. Sci. Eng. Technol. 2017, 4, 187-194.

24. Papadakis, E.; Raptopoulous, R.; Koskinopoulou, K.; Maniadakis, M. On the use of vacuum technology for applied robotic systems. In Proceedings of the 2020 6th International Conference on Mechatronics and Robotics Engineering, Barcelona, Spain, 12-15 February 2020; pp. 73-77. 
25. Valencia, A.J.; Idrovo, R.M.; Sappa, A.D.; Guingla, D.P.; Ochoa, D. A 3D vision based approach for optimal grasp of vacuum grippers. In Proceedings of the 2017 IEEE International Workshop of Electronics, Control, Measurement, Signals and their Application to Mechatronics, Donostia-San Sebastian, Spain, 24-26 May 2017; pp. 1-6.

26. Baek, S.; Kim, D.-O. Effects of Z-axis suction position of vacuum gripper to box pick-up operation. In Proceedings of the 26th Winter Conference of Society for Computational Design and Engineering, Jeju, Korea, 25-27 November 2020; p. 435.

27. Baek, S.; Kim, D.-O.; Lee, S.-J.; Yu, N.-H.; Chea, S.-I. Development of air pressure measurement system of suction cups in a vacuum gripper. In Proceedings of the 21th ACIS International Semi-Virtual Winter Conference on Software Engineering, Artificial Intelligence, Networking and Parallel/Distributed Computing, Ho Chi Minh City, Vietnam, 28-30 January 2021; pp. $1-5$.

28. Baek, S.; Jeon, S.H.; Song, E.C. Real-time monitoring of a vacuum gripper pick-up operation in a box packaging process by CNN based the box surface deviation image analysis. J. Korean Inst. Ind. Eng. 2020, 46, 107-113. [CrossRef]

29. Saufi, S.R.; Ahmad, Z.A.B.; Leong, M.S.; Lim, M.H. Challenges and opportunities of deep learning models for machinery fault detection and diagnosis: A review. IEEE Access 2019, 7, 122644-122662. [CrossRef]

30. Pillai, D.S.; Blaabjerg, F.; Rajasekar, N. A comparative evaluation of advanced fault detection approaches for PV systems. IEEE J. Photovolt. 2019, 9, 513-527. [CrossRef]

31. Baek, S. System integration for predictive process adjustment and cloud computing-based real-time condition monitoring of vibration sensor signals in automated storage and retrieval systems. Int. J. Adv. Manuf. Technol. 2021, 113, 955-966. [CrossRef]

32. Dai, X.; Gao, Z. From model, signal to knowledge: A data-driven perspective of fault detection and diagnosis. IEEE Trans. Ind. Inf. 2013, 9, 2226-2238. [CrossRef]

33. Bersimis, S.; Psarakis, S.; Panaretos, J. Multivariate statistical process control charts: An overview. Qual. Reliab. Eng. Int. 2007, 23, 517-543. [CrossRef]

34. Kazemi, P.; Giralt, J.; Bengoa, C.; Masoumian, A.; Steyer, J.-P. Fault detection and diagnosis in water resource recovery facilities using incremental PCA. Water Sci. Technol. 2020, 82, 2711-2724. [CrossRef]

35. Bencheikh, F.; Harkat, M.F.; Kouadri, A.; Bensmail, A. New reduced kernel PCA for fault detection and diagnosis in cement rotary kiln. Chemom. Intell. Lab. Syst. 2020, 204, 104091. [CrossRef]

36. Kim, J.-M.; Wang, N.; Liu, Y.; Park, K. Residual control chart for binary response with multicollinearity covariates by neural network model. Symmetry 2020, 12, 381. [CrossRef]

37. Verron, S.; Tiplica, T.; Kobi, A. Fault detection in a multivariate process with a Bayesian network. Qual. Assur. 2007, 51, 1-9. [CrossRef]

38. Namgung, K.; Yoon, H.; Baek, S.; Kim, D.Y. Estimating system state through similarity analysis of signal patterns. Sensors 2020, 20, 6839. [CrossRef] [PubMed]

39. Luo, W.; Hu, T.; Ye, Y.; Zhang, C.; Wei, Y. A hybrid predictive maintenance approach for CNC machine tool driven by Digital Twin. Rob. Comput. Integr. Manuf. 2020, 65, 101974. [CrossRef]

40. Dini, G.; Gantoni, G.; Failli, F. Grasping leather plies by Bernoulli grippers. CIRP Ann. 2009, 58, 21-24. [CrossRef]

41. Isermann, R. Fault-Diagnosis Systems; Springer: Berlin, Germany, 2006.

42. Montgomery, D.C. Design and Analysis of Experiments, 10th ed.; Wiley: Hoboken, NJ, USA, 2019.

43. Kuntoğlu, M.; Sağlam, H. Investigation of progressive tool wear for determining of optimized machining parameters in turning. Measurement 2019, 140, 427-436. [CrossRef]

44. Shinozaki, H.; Nakao, Y. Equipment comparison analysis using ANOVA of FDC statistics. In Proceedings of the 2018 International Symposium on Semiconductor Manufacturing (ISSM), Tokyo, Japan, 10-11 December 2018; pp. 1-2.

45. Naik, A.B.; Reddy, A.C. Optimization of tensile strength in TIG welding using the Taguchi method and analysis of variance (ANOVA). Therm. Sci. Eng. Prog. 2018, 8, 327-339. [CrossRef] 